\title{
Repairing the heart from within
}

\section{By Kai-Jye Lou, Staff Writer}

University College London researchers have used a thymosin $\beta 4$ peptide from RegeneRx Biopharmaceuticals Inc. to target a resident population of cardiac progenitor cells and promote tissue repair in mouse models of myocardial infarction. ${ }^{1}$ The academics now plan to screen for small molecules that target the cell population. RegeneRx could have an out-licensing opportunity for its peptide, as the company is focused on developing formulations of thymosin $\beta 4$ for indications outside of cardiovascular disease.

Myocardial infarction (MI) patients are typically prescribed drugs like $\beta$-blockers and blood thinners to help prevent further injury to an already damaged heart. However, current drugs are unable to reverse existing damage and only partly restore cardiac function.

"The usual course of a patient following an acute myocardial infarction is a gradual loss of functional cardiomyocytes and increased incidence of scarring that leads to diminished cardiac output and performance and the beginnings of progressive heart failure," said David Crockford, VP of clinical and regulatory affairs at RegeneRx.

Cell transplantation is one potential way to reverse cardiac damage and increase function post-MI. However, the approach has encountered problems in the clinic, including delivery, graft survival and graft rejection.

As an alternative, the UCL group set out to identify populations of resident cardiac progenitor cells that could be activated to proliferate and promote heart repair without the need for a cell or tissue graft.

Past studies by researchers at The University of Texas Southwestern Medical Center at Dallas showed that thymosin $\beta 4$ can protect cardiomyocytes from ischemic injury ${ }^{2}$ and promote the generation of endothelial and smooth muscle cells. ${ }^{3}$

In 2006, the UCL group found that treating quiescent adult epicardium explants with thymosin $\beta 4$ stimulated the differentiation of smooth muscle and endothelial cells. ${ }^{4}$ However, that study did not identify the progenitor cell population that gave rise to these cells, nor did it explore whether thymosin $\beta 4$ also could stimulate cardiomyocyte differentiation.

Now, the group has found a population of epicardial progenitor cells in the adult mouse heart that can be activated and characterized by the re-expression of the Wilms tumor 1 (Wt1) transcription factor. Despite its name, WT1 is ordinarily only expressed in embryonic epicardial cells and has been linked to the generation of cardiac progenitor cells during heart development. ${ }^{5}$

In a mouse model of $\mathrm{MI}$, intraperitoneal injections of thymosin $\beta 4$ increased $W t 1$ reactivation in epicardial progenitor cells and promoted a subpopulation of these progenitors to differentiate into cardiomyocytes following injury compared with injection of saline control. The resulting cardiomyocytes structurally and functionally integrated with surrounding cardiac tissue and had a mature phenotype.

In epicardial explants, almost $5 \%$ of the thymosin $\beta 4$-activated cells expressed mature cardiomyocyte differentiation markers at 14 days post-MI.

MRI analysis at 28 days post-MI showed that thymosin $\beta 4$-treated mice had smaller infarcts and significant improvements in 3 of 6 parameters of cardiac function compared with saline-treated controls ( $p \leq 0.01$ for left ventricular ejection fraction; $p \leq 0.05$ for left ventricular end diastolic volume and end systolic volume).

Results were published in Nature.

RegeneRx received exclusive, worldwide rights to thymosin $\beta 4$ from the NIH in 2001. The UCL and UT Southwestern researchers are exploring the effects of thymosin $\beta 4$ under separate material transfer agreements with the biotech and do not have IP to the peptide.

RegeneRx's RGN-352 is an injectable formulation of the thymosin $\beta 4$ peptide. In March, the FDA placed a clinical hold on a Phase II trial in acute MI patients due to cGMP compliance issues at one of the company's contract manufacturers.

RegeneRx has shifted its clinical focus to RGN-259, a topical eye drop formulation of thymosin $\beta 4$ in Phase II testing for symptomatic dry eye syndrome.

The biotech also is developing RGN-137, a topical gel formulation of thymosin $\beta 4$. The compound is in Phase II testing to treat epidermolysis bullosa.

\section{Going small}

Paul Riley, corresponding author and a professor of molecular cardiology at UCL, said the group now wants to develop screens to identify small molecules that are more efficient at stimulating cardiomyocyte differentiation than thymosin $\beta 4$.

"We suggest that there may be other molecules out there-potentially known drugs - that could be more effective than thymosin $\beta 4$ at improving the capacity of these epicardial progenitor cells to differentiate into cardiomyocytes, blood vessel cells or both," said Riley.

In addition, Riley said the group still needs to determine whether activating epicardial progenitor cells in humans will have an effect similar to that seen in mice.

Crockford said that for any molecule that promotes cardiomyocyte differentiation, it will be important to assess various doses and dosing 


\section{ANALYSIS}

regimens in both the pre- and postinjury settings, and to evaluate different routes of delivery.

"RegeneRx has synthesized a number of amino acid sequences of thymosin $\beta 4$, each displaying interesting biological activities," he added. "Possibly one of these peptides may serve in optimizing the generation of cardiomyocytes from various subpopulations of epicardial-derived progenitors."

However, Crockford also noted that thymosin $\beta 4$ 's known prosurvival and anti-inflammatory properties alone already "make it an ideal candidate for use in the MI setting."

UCL has filed a patent application covering the epicardial progenitor cells and methods for generating postnatal, epicardium-derived cells. The work is available for licensing through University College London Business plc, the university's technology transfer and commercialization arm.

RegeneRx has multiple issued and pending patents covering thymosin $\beta 4$ and its use in various indications, including MI. Crockford said the company's RGN-352 program is available for partnering or out-licensing.

"We now know that thymosin $\beta 4$ has the potential to induce the recruitment and differentiation of multipotent progenitor cells, generat- ing multilineage tissue composed of endothelium, smooth muscle and contractile myocardium. I'm not aware of any other molecule that can do these things," he said. "These findings support RegeneRx's contention that RGN-352 will play a key role in cardiovascular regenerative medicine and in regenerative medicine as a whole."

Lou, K.-J. SciBX 4(28); doi:10.1038/scibx.2011.785

Published online July 14, 2011

\section{REFERENCES}

1. Smart, N. et al. Nature; published online June 8, 2011; doi:10.1038/nature10188

Contact: Paul R. Riley, University College London, London, U.K. e-mail: p.riley@ich.ucl.ac.uk

2. Bock-Marquette, I. et al. Nature 432, 466-472 (2004)

3. Bock-Marquette, I. et al. J. Mol. Cell. Cardiol. 46, 728-738 (2009)

4. Smart, N. et al. Nature 445, 177-182 (2007)

5. Martínez-Estrada, O.M. et al. Nat. Genet. 42, 89-93 (2009)

\section{COMPANIES AND INSTITUTIONS MENTIONED}

National Institutes of Health, Bethesda, Md.

RegeneRx Biopharmaceuticals Inc. (OTCBB:RGRX), Rockville, Md. University College London, London, U.K.

University College London Business plc, London, U.K.

The University of Texas Southwestern Medical Center at Dallas, Dallas, Texas 